\title{
DESARROLLO DEL CUESTIONARIO ESPAÑOL PARA MEDIR NECESIDADES NO CUBIERTAS DE SUPERVIVIENTES DE CÁNCER (CESC)
}

\section{THE DEVELOPMENT A SPANISH QUESTIONNAIRE TO ASSESS CANCER SURVIVORS' UNMET NEEDS (CESC)}

\author{
Emilia Arrighi ${ }^{1,2}$, Albert J. Jovellit, María Dolors Navarro ${ }^{1}$ y Antoni Font ${ }^{2}$ \\ 1 Instituto Global de Salud Pública y Política Sanitaria de la Universitat Internacional de Catalunya. \\ 2 Departamento de Psicología Básica y Educacional de la Universidad Autónoma de Barcelona.
}

Resumen

Introducción: Según datos de la SEOM, existen en la actualidad más de un millón y medio de supervivientes de cáncer en España. El objetivo de este estudio es el desarrollo y evaluación de un cuestionario para medir las necesidades no cubiertas de supervivientes de cáncer.

Método: Se desarrolló y evaluó un cuestionario auto-administrado para detectar las necesidades no cubiertas de supervivientes de cáncer. Durante la Fase 1 se definió el constructo, se revisó la literatura científica y se consideró un estudio cualitativo desarrollado previamente. La propuesta del Cuestionario Español para Medir Necesidades no Cubiertas de Supervivientes de Cáncer (CESC) se evaluó sucesivamente por un panel de expertos hasta obtener una versión que incluye las propuestas formuladas. En la Fase 2, el cuestionario fue completado por un total de 109 supervivientes de cáncer, a fin de analizar sus propiedades psicométricas.

Resultados: El cuestionario CESC incluyó un total de 25 ítems o necesidades relevantes, mostrando tener buenas propiedades de fiabilidad y validez. Como resultado del análisis estadístico se obtuvieron 3 Factores: físico, emocional y laboral-económico.

Conclusiones: El cuestionario CESC es un primer instrumento disponible en español, para facilitar la detección de necesidades de servicios específicos dirigidos a la población de supervivientes de cáncer. Los resultados, si bien preliminares, son muy alentadores.

Palabras clave: Supervivientes de cáncer, necesidades no cubiertas y cuestionario.

\section{Abstract}

Background: To develop and evaluate a questionnaire to measure cancer survivors' unmet needs.

Methods: A self-report measure of cancer survivors' unmet needs was developed. In Phase I, it was taken into consideration the construct definition, literature review and previous qualitative research that identified needs in survivors. Spanish Questionnaire of Cancer Survivors' Unmet Needs (CESC) was review by an expert panel up to the final version. In Phase 2, the measure was completed by 109 cancer survivors.

Results: CESC questionnaire included 25 need items. Good acceptability, internal consistency and validity were demonstrated. Factor analysis identified three factors: Physical, Emotional and Employment/Economic.

Conclusions: The CESC, first instrument in Spanish, will facilitate the evaluation of target services and generation of service delivery recommendations for cancer survivors. Even if it is a preliminary version, results are encouraging.

Key words: Cancer survivorship, unmet needs and questionnaire. 
El cáncer es una de las principales causas de muerte a nivel mundial y constituye un tema prioritario de sanidad pública. Gracias a los avances registrados en el cribado, diagnóstico precoz y nuevos tratamientos disponibles, en las dos últimas décadas, el número de personas que han superado una enfermedad oncológica se ha triplicado ${ }^{(1)}$. También en nuestro país, los resultados en supervivencia constituyen la gran noticia de la oncología de estos tiempos, con tasas de supervivencia a cinco años de alrededor del $50 \%$ para los hombres y alrededor del $60 \%$ para el caso de las mujeres, datos que se encuentran en la media de los registrados a nivel europeo ${ }^{(2)}$. La sociedad Española de Oncología médica (SEOM) calculó que existen ya en España alrededor de un millón y medio de supervivientes de cáncer y las previsiones a nivel mundial apuntan a que las cifras registradas actualmente se duplicarán para el año $2030^{(3,4)}$.

La fase que sigue a la finalización de los tratamientos de cáncer ha sido identificada como una etapa en la que pueden observarse importantes efectos en la calidad de vida de los pacientes $y$, en este sentido, presentar necesidades especiales. Así lo ponía de relieve una publicación del Instituto Americano de Medicina( ${ }^{(5-7)}$.

Muchas personas que han finalizado los tratamientos oncológicos deben aprender a gestionar las secuelas de la enfermedad: fatiga, dolor, problemas psicológicos y emocionales, dificultades con la sexualidad y la intimidad, etc.; además de barreras en la reintegración a la vida laboral ${ }^{(7-9)}$. A ello se añade que los pacientes deben aprender a convivir con la incertidumbre de una posible recaída, y con los controles y el seguimiento clínico que no siempre se administra de manera estandarizada ${ }^{(10,11)}$.

Si bien es cierto que se han producido muchos avances en cuanto a la atención integral del paciente con cáncer, es necesario mejorar la atención a aquellas per- sonas que han finalizado los tratamientos primarios y deben continuar sus controles de seguimiento. Se debe tener en cuenta que este grupo de afectados se encuentra fuera del itinerario asistencial habitual $y$, por lo tanto, resulta más difícil que puedan contactar con los servicios que necesitan. Si bien es cierto que se han publicado distintos estudios acerca de las necesidades de supervivientes de cáncer ${ }^{(12-13)}$, no son muchos los trabajos que se han desarrollado en España ${ }^{(14-16)}$ y sólo recientemente se ha publicado un plan de seguimiento para largos supervivientes ${ }^{(17)}$.

Para identificar las necesidades no cubiertas de los supervivientes de cáncer y poder detectar qué esperan recibir los pacientes españoles de la asistencia sanitaria resulta esencial el diseño de un instrumento de fácil aplicación en la consulta clínica. La evaluación y detección de necesidades favorece la identificación de grupos que tendrían un mayor beneficio de intervenciones preventivas o de utilización de servicios específicos. En este sentido, es fundamental tener en cuenta que debido a diferencias culturales y sanitarias, las necesidades en nuestro país, no tienen porqué coincidir con los de la población para la que fueron creados los instrumentos actualmente disponibles ${ }^{(18-19)}$.

Así pues, el objetivo principal de este estudio es la construcción de un cuestionario auto-administrado (CESC) para evaluar las necesidades no cubiertas de supervivientes de cáncer (Fase 1); y valorar las propiedades psicométricas del instrumento (Fase 2).

\section{MÉTODO}

\section{Fase 1: Diseño del cuestionario}

El desarrollo del cuestionario CESC se ha basado en (a) definición del constructo; (b) revisión de la literatura científica sobre el tema y de instrumentos de medida que respondieran a este constructo; (c) revisión 
de los resultados del estudio cualitativo previo para conocer las necesidades de supervivientes de cáncer en España ${ }^{(14)}$.

a. Para definir el constructo se ha considerado la descripción hecha por el Instituto Americano del Cáncer que incluye a la persona afectada y a los familiares ${ }^{(20)}$.

b. La revisión de la literatura científica incluyó la identificación de los instrumentos disponibles, considerándose como más adecuados para la detección de necesidades el "Cancer Survivors' Unmet Needs measure"(21); el "Supportive Care Needs Survey"(22) y el "Lance Armstrong Foundation Sur$v^{\prime} y^{\prime \prime 23)}$, que se tomaron como punto de referencia para diseñar los ítems.

c. El estudio cualitativo incluyó la realización de nueve entrevistas semiestructuradas con expertos considerados informadores clave, representantes de las sociedades científicas nacionales del ámbito de la oncología y organizaciones de pacientes de ámbito nacional. Además, se realizaron 3 grupos de discusión con pacientes supervivientes de cáncer y uno con familiares por un total de 20 participantes. Los criterios de inclusión consideraron a personas mayores de 18 años, que habían finalizado tratamiento oncológico al menos 2 años antes y que estuvieran interesados en participar. Los participantes se segmentaron por edad en tres grupos (18-40, 41-60 y mayores de 60 años), en base a una muestra teórica que incluyó supervivientes de distintos tipos de cáncer (mama, ginecológico, hematológico y colon y recto).

\section{Construcción del de cuestionario}

A partir del estudio cualitativo y la revisión de otros instrumentos se elaboró un primer cuestionario auto-administrado compuesto por 25-item distribuidos en 4 dominios: necesidades físicas, necesidades emocionales, necesidades sociales y necesidades económicas y laborales. A los participantes se les solicitó primero que indicaran, desde que habían finalizado el tratamiento, (a) 'si tenían alguna necesidad en cada dominio' y en el caso de que hubieran recibido algún tipo de asistencia o consejo pudieran valorar (b) 'qué grado de satisfacción tenían en base a la asistencia recibida' (puntuando en una escala desde 'muy satisfecho, algo satisfecho, satisfecho o nada satisfecho'). Se les pidió además que identificaran necesidades de información y formación eligiendo entre una lista de seis ítems entre los que se mencionan la incorporación de una dieta saludable, la inclusión de una rutina de ejercicio físico o el manejo de técnicas para controlar las emociones. También se les consultó acerca de la importancia de poder contar con un Plan de Seguimiento al Superviviente considerando 'la posibilidad de ser atendidos por parte de un equipo interdisciplinario', 'ser atendidos en unidades específicas', 'disponer de un teléfono de consulta' o 'participar en la toma de decisiones en la fase posterior al tratamiento'. Para recoger esta información se solicitó a los participantes que marcaran el nivel de importancia (muy necesario, necesario, poco necesario y nada necesario). El instrumento incluyó además una sección de datos socio-demográficos e información acerca del estado de salud. Los pacientes necesitaron entre 10 y 15 minutos aproximadamente para contestar el cuestionario.

\section{Valoración de la primera versión del cuestionario}

Una vez construida una primera versión, el instrumento fue evaluado por un panel de expertos integrado por 2 mé- 
dicos generales, 2 oncólogos, 2 psicooncólogos, un grupo de enfermeras oncológicas de un gran hospital de ciudad y 4 líderes de organizaciones de pacientes. Los mismos evaluaron el grado de comprensión, oportunidad de los ítems, longitud y diseño del cuestionario, efectuando observaciones que se tomaron en consideración y proponiendo modificaciones mínimas. El objetivo de esta revisión fue la de poder someter a juicio el cuestionario por parte de profesionales de amplia experiencia en la atención de pacientes con distintas patologías oncológicas, además de la visión y perspectiva única de los mismos afectados ${ }^{(24)}$.

Fase 2: Estudio empírico para valorar las propiedades psicométricas del CESC

\section{Procedimiento}

El reclutamiento de los participantes para la segunda muestra (o muestra para el análisis psicométrico) se realizó durante el VI Congreso Español de Pacientes con Cáncer organizado por GEPAC (Grupo Español de Pacientes con Cáncer) que tuvo lugar en Madrid el 26 y 27 de noviembre de 2011. Los asistentes al congreso, provenientes de distintos lugares de España, fueron informados acerca delos objetivos del estudio e invitados a participar voluntariamente en el mismo. Los criterios de inclusión fueron: pacientes mayores de 18 años, diagnosticados de patología oncológica, que hubieran finalizado los tratamientos al menos un año antes de la participación en el estudio, actualmente libres de enfermedad y que estuvieran interesados en participar.

\section{Análisis estadístico}

El análisis estadístico del CESC se realizó utilizando el programa SPSS 17. El análisis factorial ha sido realizado por el método de componentes principales y rotación Varimax. Para la extracción del número de factores se han tenido en cuenta principalmente los criterios de parsimonia e interpretabilidad de los mismos ${ }^{(25-26)}$, juntamente con el análisis paralelo. También se han considerado la magnitud de los valores propios, el porcentaje de varianza explicado por el modelo y los valores de las correlaciones residuales. Para la asignación de los ítems a las componentes, se han utilizado cargas factoriales superiores a 0,3 .

\section{RESULTADOS}

\section{Participantes}

Se recogieron un total de 118 cuestionarios; 9 fueron descartados porque no cumplían con los criterios de inclusión establecidos y/o se encontraban incompletos. Así pues, la muestra final estuvo formada por 109 personas, con una media de edad de 52,7 años (Desviación Típica de 12,1$)$. Más del $80 \%$ habían finalizado los tratamientos oncológicos entre uno y cinco años atrás. Las características sociodemográficas de la muestra se presentan en la tabla 1.

Se aplicó un Análisis en Componentes Principales para explorar la estructura interna del cuestionario en la parte correspondiente a los ítems relativos a las necesidades para cada dimensión, para observar su agrupamiento y poder así simplificar su interpretación. La prueba de esfericidad de Bartlett resultó significativa $\left(\mathrm{X}^{2}{ }_{300}=677\right.$; $\mathrm{p}<0,01)$ y se obtuvo un valor de KMO de 0,719 , valor suficientemente alto para el análisis de estos datos, así como un determinante de la matriz de correlaciones de 0,001 .

Tras la primera aplicación, tal como mostró el gráfico de sedimentación, se obtuvieron 8 componentes con valor propio superior a 1, de los cuales sólo 3 obtenían 
Tabla 1. Características socio-demográficas de la muestra (n \%)

\begin{tabular}{|c|c|c|}
\hline \multirow{2}{*}{$\begin{array}{l}\text { Variable } \\
\text { Género }\end{array}$} & \multicolumn{2}{|c|}{$n=109 \%$} \\
\hline & & \\
\hline Masculino & 32 & 29,4 \\
\hline Femenino & 77 & 70,6 \\
\hline \multicolumn{3}{|l|}{ Estado civil } \\
\hline Soltero/a & 24 & 22 \\
\hline Casado/a & 72 & 66 \\
\hline Viudo/a & 3 & 2,8 \\
\hline Separado/a o Divorciado/a & 10 & 9,2 \\
\hline \multicolumn{3}{|l|}{ Educación } \\
\hline Estudios primarios & 11 & 10,1 \\
\hline Estudios secundarios & 38 & 34,8 \\
\hline Estudios superiores o universitarios & 55 & 50 \\
\hline No responde & 5 & 5,1 \\
\hline \multicolumn{3}{|l|}{ Empleo } \\
\hline Empleado por cuenta propia o ajena & 40 & 36,7 \\
\hline En paro & 12 & 11 \\
\hline Jubilado & 21 & 19,3 \\
\hline Incapacidad & 14 & 12,8 \\
\hline Estudiante & 2 & 1,8 \\
\hline Otro & 20 & 18,4 \\
\hline \multicolumn{3}{|l|}{ Nivel de ingresos totales anuales (en euros) } \\
\hline Menos de 12.000 & 27 & 24,8 \\
\hline De 12.001 a 24.000 & 25 & 22,9 \\
\hline De 24.001 a 36.000 & 19 & 17,4 \\
\hline Más de 36.000 & 25 & 23 \\
\hline No responde & 13 & 11,9 \\
\hline \multicolumn{3}{|c|}{ Tiempo desde que finalizaron los tratamientos (en años) } \\
\hline 1 a 5 & 89 & 81,7 \\
\hline 6 a 10 & 8 & 7,3 \\
\hline Más de 10 & 12 & 11 \\
\hline \multicolumn{3}{|l|}{ Tipo de cáncer } \\
\hline Hematológico & 42 & 38,5 \\
\hline Mama & 36 & 33 \\
\hline Otro & 31 & 28,4 \\
\hline \multicolumn{3}{|l|}{ Tipo de tratamiento* } \\
\hline Quimioterapia & 87 & 79,8 \\
\hline Radioterapia & 50 & 45,9 \\
\hline Cirugía & 59 & 54,1 \\
\hline Trasplante de médula & 13 & 11,9 \\
\hline Otro & 18 & 16,5 \\
\hline \multicolumn{3}{|l|}{ Co-morbilidad } \\
\hline Obesidad & 14 & 12,8 \\
\hline Hipertensión & 16 & 14,7 \\
\hline Problemas óseos & 20 & 18,3 \\
\hline Diabetes & 4 & 3,7 \\
\hline Enfermedades cardiovasculares & 8 & 7,3 \\
\hline Otras & 25 & 22,9 \\
\hline
\end{tabular}

*Algunos participantes han realizado distintos tratamientos. 
un valor 1,5 o más. Mediante un Análisis Paralelo, se obtuvieron valores propios inferiores al promedio de 100 simulaciones en las tres primeras componentes. En el gráfico de sedimentación, se observan cambios de pendiente en la recta del gráfico a partir de la primera componente y a partir de la cuarta principalmente. El porcentaje de varianza acumulado por las 3 primeras componentes fue del $35,77 \%$, el $41,4 \%$ en el caso de 4 componentes y el $46,59 \%$ en el caso de 5 componentes. Las soluciones de 1 y 2 componentes explican menos del $30 \%$ de la varianza por lo que se rechazaron estas soluciones.

Al aplicar la solución de 3 factores, el valor residual (diferencia entre correlaciones reproducidas y originales) máximo obtenido en valor absoluto fue de 0,21 , con un promedio de 0,06 , con el $78,4 \%$ con valores inferiores a 0,1 . Las soluciones de 4 y 5 factores no mejoraban significativamente estos índices, ni la interpretación de las componentes escogidas, por lo que se decidió por la solución de 3 factores. En cuanto a las comunalidades, oscilaron entre 0,14 y 0,59, excepto para el ítem p9 que correspondía a la categorización "otros síntomas".

Inicialmente se aplicó una rotación oblimin (con valor delta 0) para analizar la relación entre las componentes. Se obtuvo una correlación máxima de 0,242 entre la primera y la segunda componentes, 0,195 entre la primera y la tercera, y 0,123 entre las dos últimas. Además las matrices de configuración y de estructura presentaban soluciones muy parecidas por lo que se decidió interpretar la matriz rotada con el método varimax (rotación ortogonal) de manera que se facilitase la interpretación de las componentes.

La matriz de cargas factoriales rotadas se muestra en la tabla 2.

El Factor 1 sobre necesidades emocionales agrupó las necesidades relativas a tristeza, depresión, conductas de evitación social, preocupación por el aspecto físico, por la muerte y por las recaídas, ansiedad, angustia, además de la relación de pareja y conductas de evitación social. El Factor 2 sobre necesidades físicas, incluyó las personas con puntuaciones altas que presentaron problemas con el desplazamiento, dolor y malestar general, problemas para dormir, sensación de hormigueo, pérdida de energía y cansancio, problemas de visión. El Factor 3 sobre problemas en el ámbito laboral y económico principalmente producido por los efectos de la enfermedad.

Utilizando el método de ponderación simple para el cálculo de las puntuaciones factoriales, se observaron correlaciones entre los resultados y las puntuaciones factoriales obtenidas mediante el ACP de 0,934 para el factor 1, 0,922 para el factor 2 , y 0,941 para el tercero, con lo que las puntuaciones en las 3 componentes mediante el ACP y las obtenidas mediante la suma de las puntuaciones de los ítems con cargas factoriales superiores a 0,3 son análogas, y por simpleza de cálculo, es preferible trabajar con las puntuaciones resultantes de la suma simple de ítems.

Respecto a la fiabilidad de las 3 componentes, los valores alfa de Cronbach obtenidos han sido de 0,775 para la primera componente, 0,713 para la segunda, y 0,636 para la tercera, considerados como aceptables en las primeras fases de construcción de un test diseñado para los objetivos citados, y teniendo en cuenta el número de ítems de cada componente (9, 8 y 7), así como la heterogeneidad de su contenido, sobre todo de la componente 3. En la tabla 3 se muestra la matriz de correlaciones.

\section{Necesidades identificadas}

La frecuencia de las necesidades más prevalentes se muestran en la Tabla 4. Alrededor del $80 \%$ de los participantes ha 


\section{Tabla 2. Matriz de cargas factoriales rotadas}

\begin{tabular}{|c|c|c|c|}
\hline \multirow[b]{2}{*}{ Ítem } & \multicolumn{3}{|c|}{ Componente } \\
\hline & 1 & 2 & 3 \\
\hline pg9_c Relación de pareja perjudicada o interrumpida &, 707 & & \\
\hline pg9_d Tristeza y depresión & ,704 & & \\
\hline pg9_a He evitado conocer nuevas personas &, 677 & & \\
\hline pg9_e No me he sentido apoyado por mi pareja &, 641 & & \\
\hline pg9_b Menos ganas de participar en reuniones sociales & 602 & & \\
\hline pg6_d Preocupación muerte &, 549 & & \\
\hline pg6_e Preocupación cambios aspecto físico &, 468 &, 369 & \\
\hline pg6_b_2 Angustia, ansiedad, preocupación, pérdida de esperanza &, 442 & & \\
\hline pg9_f Dificultades en relaciones sexuales &, 357 & & \\
\hline pg3_o Problemas para desplazarme &, 265 &, 640 & \\
\hline pg3_e Dolor o malestar general & &, 632 & \\
\hline pg3_n Problemas para dormir & &, 599 & \\
\hline pg3_d Poca sensibilidad o sensación de hormigueo & &, 594 & \\
\hline pg3_a Pérdida de energía y cansancio & &, 586 & \\
\hline pg3_h Problemas de visión & &, 561 & \\
\hline pg6_a Miedo a recaída & &, 338 & \\
\hline pg3_b Pérdida de memoria y concentración & & ,330 & \\
\hline pg9_i Otros & &, 218 &,- 213 \\
\hline pg12_c No puedo trabajar igual que antes & & & ,708 \\
\hline pg12_f Problemas de reincorporación al trabajo & &, 344 & 609 \\
\hline pg12_i Problemas económicos & & &, 588 \\
\hline pg12_k_cOtros problemas con el trabajo y/o economía & & &, 566 \\
\hline pg12_e La enfermedad ha sido un obstáculo para promoción profesional &, 321 & &, 501 \\
\hline pg12_a He perdido trabajo & & & 416 \\
\hline pg3_r_zOtros & & &, 362 \\
\hline
\end{tabular}

Factor loading $\geq 0,3$ 


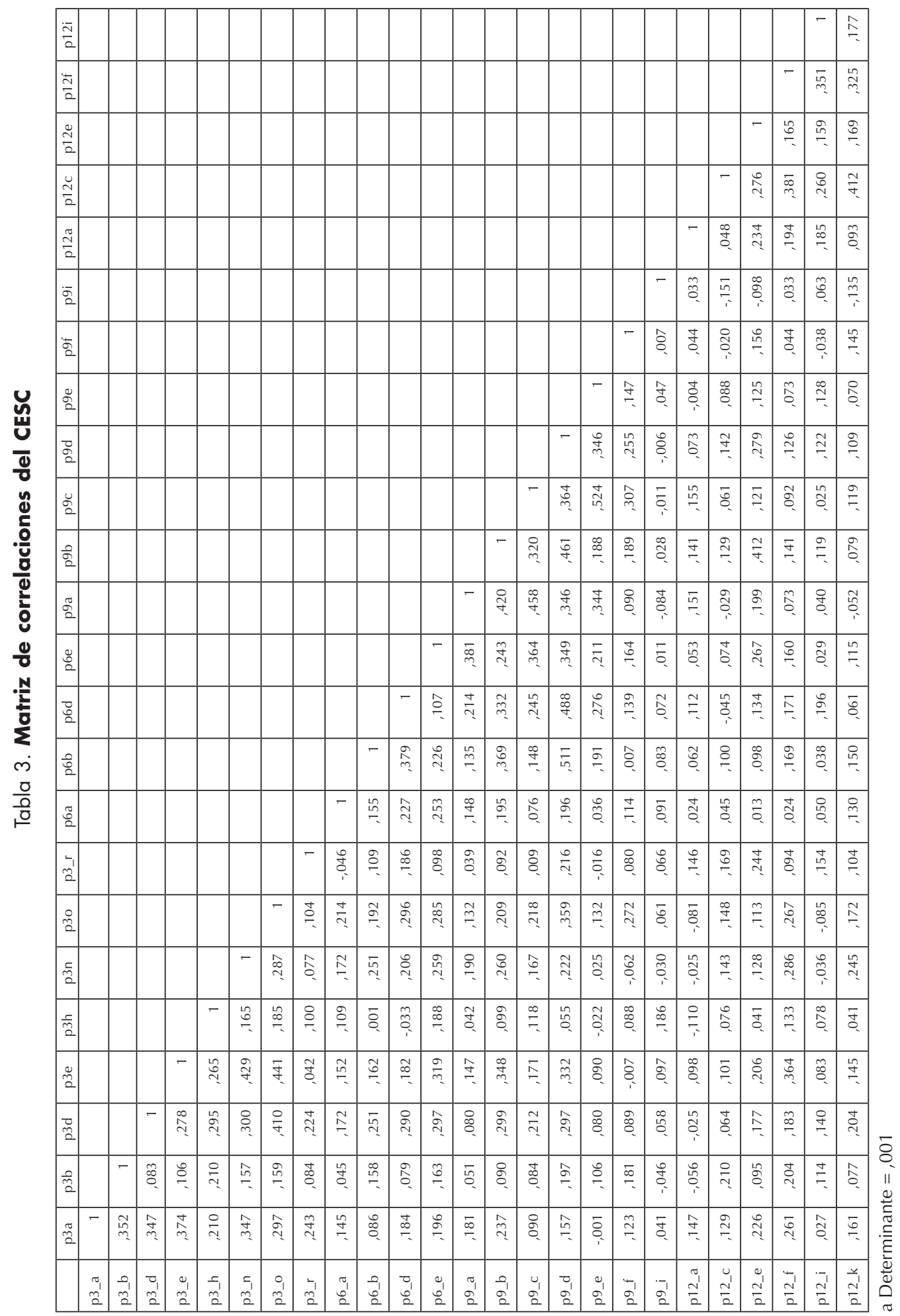


referido al menos una necesidad no cubierta. Entre las dimensiones exploradas, los participantes al estudio han mostrado una mayor frecuencia de necesidades no cubiertas en relación al área emocional y física, siendo menores las necesidades económicas y laborales.

\section{DISCUSIÓN}

El objetivo de este estudio fue el de desarrollar y evaluar un instrumento para medir las necesidades no cubiertas de supervivientes de cáncer. Este estudio aporta evidencia que el cuestionario CESC reúne los estándares metodológicos relativos a la validez y fiabilidad necesarias para un instrumento en su primera versión. Al ser un instrumento auto-administrado y fácil de cumplimentar, puede ser incorporado a la práctica clínica, para la identificación de necesidades no cubiertas de esta población o necesidades que pueden cambiar a lo largo del tiempo.

Al no existir un registro nacional de supervivientes en España, ni estudios previos de este tipo, no se ha podido disponer de una población de referencia; por lo que estos resultados se contrastarán con resultados de cuestionarios que miden el mismo constructo que se han realizado en otros países. En este sentido, alrededor del $80 \%$ de los entrevistados declara al menos una necesidad no cubierta en el periodo posterior a los tratamientos, en congruencia con otros estudios que reportan valores cercanos a los 2/3 de los consultados $^{(21-22)}$. Más del $50 \%$ de los entrevistados declaró la necesidad de recibir mayor información durante la etapa de superviviencia, además de consejo para la incorporación de una dieta saludable y de técnicas de manejo de las emociones, resultados similares a los publicados por Absolom y Zebrack ${ }^{(27,28)}$. Entre las necesidades no cubiertas más prevalentes se encuentran la pérdida de energía, el miedo a una posible recaída y sentimientos de angustia, ansiedad y preocupación acerca del futuro, datos avalados por otros estudios $^{(29,30)}$.

Entre los aportes del presente estudio se quiere destacar que se trata de un estudio pionero para esta población. Además, las características de la muestra han puesto en evidencia las necesidades de los supervivientes de edad menor de 40 años, menos estudiada y más propensa a presentar necesidades no cubiertas ${ }^{(28,29,31)}$. Además, la mayoría de los encuestados presenta problemas a nivel de la reinserción laboral y que ninguno de los encuestados recibió asistencia para poder resolver estos problemas, cuestión que resulta de interés y estudio a nivel internacional|(32-33).

A los participantes se les consultó además acerca de la importancia de disponer de un Plan de Seguimiento Estandarizado y el $80 \%$ ha considerado 'importante' o 'muy importante' poder ser atendido por un equipo interdisciplinario que incluya la presencia de un psicólogo y trabajador social, ser atendidos por servicios específicos, disponer de un teléfono de consulta y tener una copia del plan de tratamientos y participar activamente en las decisiones que le afectan. Esta estrategia que ya ha sido implementada en otros países como recurso para la identificación de necesidades de supervivientes sería nueva en nuestro país ${ }^{(34,35)}$.

Entre las limitaciones principales del presente estudio se debe mencionar que la muestra ha sido de conveniencia y que los participantes, en su mayoría, pertenecían a organizaciones de pacientes. Es necesario realizar estudios que puedan confirmar estos resultados con muestras más amplias y que aporten aún mayor representatividad.

\section{CONCLUSIONES}

Este estudio aporta evidencia acerca de la presencia de necesidades no cubiertas 
Tabla 4. Mayor frecuencia de necesidades no cubiertas por dominio (n \%)

\begin{tabular}{clcc}
\hline Posición & Ítem & $n(\%)$ & Dominio \\
\hline 1 & $\begin{array}{l}\text { Pérdida de energía y cansancio la mayor } \\
\text { parte del día }\end{array}$ & $78(71,6)$ & Necesidades físicas \\
2 & Miedo a una posible recaída & $65(59,6)$ & Necesidades emocionales \\
3 & Angustia, ansiedad y preocupación & $65(59,6)$ & Necesidades emocionales \\
4 & Pérdida de memoria y concentración & $55(50,5)$ & Necesidades físicas \\
5 & Problemas con la sexualidad & $51(46,8)$ & Necesidades físicas \\
6 & Problemas para dormir & $51(46,8)$ & Necesidades físicas \\
7 & Sentimientos de tristeza y depresión & $45(41,3)$ & Necesidades emocionales \\
8 & Preocupación por los cambios en el & $41(37,6)$ & Necesidades emocionales \\
9 & aspecto físico & $37(33,9)$ & Necesidades sociales \\
& Disminución en la participación en & reuniones sociales & Necesidades laborales y \\
10 & No poder trabajar igual que antes & $35(32,1)$ & económicas \\
\hline
\end{tabular}

en la población de pacientes que han finalizado tratamientos oncológicos. Entre las más prevalentes se encuentran las necesidades de tipo psicológico, que con frecuencia son subestimadas o poco consideradas en la rehabilitación de los pacientes.

Es necesario un cribado sistemático de la población de supervivientes de cáncer para que, mediante la derivación a los servicios correspondientes, sea posible una recuperación completa, tanto física como psicosocial. Este instrumento puede constituir un recurso esencial a la hora de identificar cuáles son las necesidades de esta población y resulta de especial interés visto que la SEOM ha apenas publicado un protocolo para el seguimiento de los largos supervivientes de cáncer en España ${ }^{(17)}$.

\section{AGRADECIMIENTO}

Los autores desean agradecer al Foro Español de Pacientes y al Grupo Español de Pacientes con Cáncer (GEPAC) por la colaboración en el reclutamiento de los candidatos que han participado en la encuesta; así como también a los profesionales que han revisado el cuestionario y han aportado sus sugerencias.

\section{REFERENCIAS BIBLIOGRÁFICAS}

1. Globocan 2012. En línea. [Acceso 1 de marzo de 2013]. Disponible en: http:// globocan.iarc.fr/factsheets/populations / factsheet.asp? uno $=900_{-}$

2. Sant M, Allemani C, Santaquilani M, Knijn A, Marchesi F, Capocaccia R; EUROCARE Working Group. Survival of cancer patients diagnosed in 1995-1999. Results and commentary. Eur J Cancer 2009;45:93191. Doi: 10.1016/j.ejca.2008.11.018.

3. Ries LAG, Melbert D, Krapcho M, Mariotto A, Miller B, Feuer E, et al. SEER Cancer Statistics Review, 1975-2004, National Cancer Institute. Bethesda, MD. En línea. [Acceso 1 de enero de 2012]. Disponible en: http://seer.cancer.gov/csr/1975_2004/.

4. Sociedad Española de Oncología Médica. El cáncer en España, 2012. En línea. [Acceso 1 de febrero de 2013]. Disponible 
en: http://www.seom.org/es/prensa/el-cancer-en-espanyacom?format=pdf.

5. Earle, CC. Failing to plan is planning to fail: Improving the quality of care with survivorship care plans. J Clin Oncol 2006;24:5112-6.

6. Ganz PA, Casillas J and Hahn E. Ensuring quality care for cancer survivors: Implementing the survivorship care plan. Sem Onc Nur 2008;24:208-17. Doi: 10.1016/j. soncn.2008.05.009

7. Hewitt M, Ganz P, editors. From cancer patient to cancer survivor-lost in transition: An American Society of Clinical Oncology and Institute of Medicine Symposium. National Academies Press, Washington, DC; 2000.

8. Ganz, PA. A teachable moment for oncologists: Cancer survivors, 10 million strong and growing. J Clin Oncol 2005;23:545860.

9. Jacobs LA, Palmer SC, Schwartz LA, DeMichele A, Mao JJ, Carver J, et al. Adult cancer survivorship: Evolution, research, and planning care. Ca J for Clin 2009;59:391410. Doi: 10.3322/caac.20040.

10. Feliu J, Virizuela JA. El seguimiento en los supervivientes de cáncer: una responsabilidad compartida. Med Clín (Barc) 2011;137:163-5. Doi: 10.1016/j.medcli.2011.03.016

11. Ferro T and Borràs J.M. Una bola de nieve está creciendo en los servicios sanitarios: los pacientes supervivientes de cáncer. Gac Sanit 2011;25:240-5. Doi: 10.1016/j. gaceta.2010.12.002

12. Jacobsen, P.B. Clinical practice guidelines for the psychosocial care of cancer survivors. Cancer 2009;115(S18): 4419-29. Doi: 10.1002/cncr.24589

13. Rowland $\mathrm{JH}$, Hewitt $M$ and Ganz PA. Cancer survivorship: A new challenge in delivering quality cancer care. J Clin Oncol 2006;24: 5101-4. Doi: 10.1200/ JCO.2006.09.2700

14. Arrighi E, Jovell AJ. and Navarro Rubio, MD. Después del cáncer. Necesidades y experiencias de personas que han superado una enfermedad oncológica. Fundación Josep Laporte Eds., Barcelona; 2011.

15. Buscemi V, Font A, Viladrich C. Focus on relationship between the caregivers unmet needs and other caregiving outcomes in cancer palliative care. Psicooncología 2010;7:109-25.

16. Solana, AC. Aspectos psicológicos en el paciente superviviente. Oncología 2005;28:157-63.

17. Sociedad Española de Oncolcogía Médica. Largos supervivientes de cáncer, 2013. En línea. [Acceso 1 de octubre de 2013]. Disponible en: http://www.seom. org/seomcms/images/stories/recursos/infopublico/publicaciones/MONOGRAFICO_ SEOM_LARGOS_SUPERVIVIENTES_I.pdf_

18. Arrighi E, Jovell A.J. and Navarro M.D. El valor terapéutico en Oncología. La perspectiva de pacientes, familiares $y$ profesionales sanitarios. Psicooncología 2010;7:363-74.

19. Jovell AJ, Navarro Rubio, Fernández Maldonado L, Blancafort S. Nuevo rol del paciente en el sistema sanitario. Aten Primaria 2006;38:234-7. Doi: 10.1157/13092347

20. National Cancer Institute. Transitional Care Planing, 2002. En línea. [Acceso 1 de diciembre de 2012] Disponible en: http:// www.cancer.gov/cancertopics/pdq/supportivecare/transitionalcare/Patient/page2

21. Hodgkinson K, Butow P, Hunt GE, Pendlebury S, Hobbs KM, Lo SK, Wain, G. The development and evaluation of a measure to assess cancer survivors> unmet supportive care needs: The CaSUN (Cancer Survivors> Unmet Needs measure). Psychooncology 2007;16:796-804. Doi: 10.1002/pon.1137

22. Boyes A, Girgis A, Lecathelinais C. Brief assessment of adult cancer patients' perceived needs: Development and validation of the 34-item Supportive Care Needs Survey (SCNS-SF34). J Eval Clin Prac 2009;15:602-6. Doi: 10.1111/j.13652753.2008.01057 
23. Lance Armstrong Foundation Survey 2010 y 2012. En línea. [Acceso 1 de junio de 2012]. Disponible en: http://www.surveygizmo. com/s3/936386/LIVESTRONG2012?s_ $\mathrm{src}=$ blog\&s_subsrc $=1$

24. Viladrich Segués M, Doval Diéguez E, Prat Santolària R. Vall-Ilovera, M. Psicometría. Barcelona: Ediciones UOC; 2005.

25. Abad FJ, Olea J, Ponsoda V, García C. Medición en Ciencias sociales y de la salud. Madrid: Síntesis; 2001.

26. Gorsuch, R. Factor analysis. Ed. L. Erlbaum Associates, 2nd Edition, New York, 1983.

27. Absolom K, Eiser C, Michel G, Walters S, Hancock B, Coleman R, Snowden J, Greenfield D. Follow-up care for cancer survivors: Views of the younger adult. Br J Cancer 2009;18;101:561-7. Doi: 10.1038/sj.bjc.6605213

28. Zebrack BJ, Ganz PA, Bernaards CA, Petersen L, Abraham L. Assessing the impact of cancer: Development of a new instrument for long-term survivors. Psychooncology 2006;15:407-21. Doi: 10.1002/pon.963

29. Boyes A, Girgis A, D'Este CA, Zucca AC. Prevalence and correlates of cancer survivors' supportive care needs 6 months after diagnosis: A population-based crosssectional study. BMC Cancer 2012;12:150 Doi:10.1186/1471-2407-12-150.

30. Knobf MT, Ferrucci LM, Cartmel B, Jones BA, Stevens D Smith $M$, et al. Needs assessment of cancer survivors in Connecticut. J Can Surv 2012;6:1-10. Doi: 10.1007/ s11764-011-0198-2

31. McDowell $M$, Occhipinti S, Ferguson $M$, Chambers S. Predictors of change in unmet supportive care needs in cancer. Psychooncology 2010;19:508-16. Doi: 10.1002/pon.1774

32. de Boer AGEM, TaskilaT, Ojajärvi A, van Dijk $\mathrm{FJH}$ and Verbeek JHAM. Cancer survivors and unemployment. JAMA 2009;301:75362. Doi: 10.1001/jama.2009.187

33. Taskila T, Lindbohm M.L. Factors affecting cancer survivors' employment and work ability. Acta Oncol 2007;46:446-51. Doi: 10.1080/02841860701355048)

34. ASCO-ESMO consensus statement on quality cancer care. Ann Oncol 2007;17:10634. Doi: 10.1093/annonc/mdl152

35. LIVESTRONG Care Plan, 2004. En línea. [Acceso el 1 de diciembre de 21012]. Disponible en: http://es.oncolink.org/oncolife/questions.cfm?es=1 